\title{
THE DEVELOPMENT OF AN EXPERT SYSTEM FOR MEASUREMENT SYSTEMS EVALUATION
}

\author{
Jiin Wang, Alabama State University, jwang@alasu.edu
}

\begin{abstract}
This article examines and demonstrates the use of expert systems (ES) technology to augment and to enhance measurement systems evaluation procedures. It presents the results of a research project in which an expert system that utilizes a knowledge-based methodology to facilitate or to automate the process in conducting a gauging $R \& R$ analysis is developed. The paper begins with a discussion of the steps involved in a variable gauge study, pointing out that inefficiencies and inaccuracies often arise due to the lengthy time requirement and heavy statistical burden placed on the study team. The paper then describes the features and the functionality of the $P C$-based expert system developed in the research. Numerous screens are provided to illustrate these features. Finally, a synopsis of the primary benefits of the system is given, as well as its limitations that call for further research efforts.
\end{abstract}

Keywords: Information Technology (IT), Expert Systems, Knowledge-based Systems, R\&R Study, Gauge Capability, Statistical Process Control

\section{INTRODUCTION}

Attainment of quality in production requires the performance of a wide variety of identifiable quality tasks throughout the manufacturing environment. An essential practice usually employed to achieve the quality assurance tasks is to conduct a manufacturing systems qualification study of a particular machine or process. Manufacturing systems qualification is an essential way of ensuring that the job is done right the first time. This is important especially for manufacturing firms who have adopted "lean" manufacturing strategies such as just-in-time (JIT) production scheduling [3].

The manufacturing systems qualification study is often performed as a team effort. In order to provide the necessary expertise, the team conducting the study is usually composed of members from four different functional areas: product engineering, production engineering, manufacturing, and quality assurance [7]. The specific structure and design of a given manufacturing systems qualification study can vary substantially depending on management's objectives, the environment, and end uses of the study. However, the prerequisite for such a study is always the assessment of the measurement systems involved.

The measurement systems evaluation is to evaluate a measuring device with respect to certain attributes of gauging, such as accuracy, stability, repeatability, and linearity [6]. This analysis will be used by the manufacturing systems qualification team to verify that the measuring equipment to be used in the machine qualification study and foregoing process control is capable of accomplishing the required measurement function.

The measurement systems evaluation expert system to be implemented in the research will fulfill this need. The systematic approach utilized in the expert system could lead to substantial increases in productivity of the responsible machine/process qualification staff, which should also lead to reduced costs in conducting a qualification study. In the form of an expert system, the statistical expertise required to effectively accomplish a comprehensive measurement system evaluation study will become an automatic part of the process. This would allow the qualification program to achieve its full potential in the overall quality assurance strategy.

\section{METHODOLOGY AND DEVELOPMENT}

\section{Overview of Measurement Systems Evaluation}

A measurement systems evaluation study is a powerful tool to assess and characterize the gauging equipment to be used in ongoing quality assurance and process control. Because gauging is subject to variation, an analysis of a process cannot be meaningful unless the measurement systems used to collect data are both accurate and stable. The measurement must be able to be made by different individuals (reproducibility) and repeated by the same person measuring the same measurement (repeatability). Therefore, a variable gauge study is usually performed in determining the gauge repeatability and reproducibility (R\&R).

According to General Motors, a formal definition to these two terms is as follows: 
Gauge Repeatability is the variation in measurements obtained when one operator uses the same gauge for measuring the identical characteristics of the same parts.

Gauge Reproducibility is the variation in the average of measurements made by different operators using the same gauge when measuring identical characteristics of the same parts [5].

In practice, there are two forms of gauging study: short method and long method. Depending on the nature of the manufacturing operation, either or both of the procedures should be set up to assist the quality engineers in reviewing gauging issues before a machine/process qualification assessment is made. The short method provides a quick and meaningful way of determining the acceptability of gauge variation. However, one disadvantage of this method is that gauge repeatability and reproducibility cannot be isolated. The results reflect a combination of both types of gauge error. The long method, on the other hand, can determine errors of gauge repeatability and reproducibility separately. The study results can also provide information concerning the possible causes of gauge error.

The evaluation process is a complex, knowledge intensive and time-consuming task. It is necessary to have a well-defined and structured guideline to lead the study team of quality engineers in performing such an evaluation. At the present, unfortunately, the team members must rely heavily on their own knowledge of statistical process control and on expertise gained over years of working experience simply because this study has yet to be automated.

A general framework for conducting a gauging $\mathrm{R} \& \mathrm{R}$ study is structured in this research. This methodology, the decision rules and heuristics, is then transformed into a knowledge base and incorporated in the measurement systems evaluation expert system.

\section{Construction of the Expert System}

An expert system is a computing system that encodes and embodies organized knowledge of a specialized domain. The measurement systems evaluation expert system is developed to be such a knowledge-based system that will facilitate a gauging R\&R study. This section presents the framework for the expert system, a discussion of its architecture that includes the theoretical concepts and implementation techniques employed.

In expert system development, an important issue is the choice of expert system implementation language or environment. Most early expert systems were written in conventional AI languages such as LISP or PROLOG. More recently, with the availability of a wide variety of commercial expert system shells, the trend is switched to the use of pre-written inference engines such as CLIPS [2] and EXSYS [4]. The measurement systems evaluation expert system, however, is written entirely in the $\mathrm{C}++$ programming language for efficiency and flexibility. The rationale of using $\mathrm{C}++$ as the vehicle for implementing this expert system is the fact that $\mathrm{C}++$ has several unique features that make it the language of choice for modern expert system development. Two of the more valuable features are dynamic memory allocation capability and the support of pointers. Both of the techniques were heavily utilized in the construction of the expert system's backward-chaining inference engine, the rule parser, and the working memory.

\section{Knowledge Base Implementation}

Several conventions for representing knowledge have been suggested in expert systems literature. The pros and cons of the different representation schemes are still a source of controversy among theoreticians. In the measurement systems evaluation expert system, the knowledge base consists of sets of rules as premise-action pairs in the basic form: IF [condition] THEN [conclusion]. They are principally used in the encoding of empirical associations between patterns of data presented to the system and actions that the system should perform consequently. For example:

$$
\begin{array}{ll}
\text { IF } & P_{1} \text { AND } \ldots \text { AND } P_{n} \\
\text { THEN } & A_{1} \text { AND } \ldots \text { AND } A_{n}
\end{array}
$$

with the reading "if premises $P_{1}$ and $\ldots$ and $P_{n}$ are true, then perform actions $A_{1}$ and ... and $A_{n}$."

In the expert systems literature, a condition is usually coded as an "object-attribute-value" triple and a conclusion clause is expressed simply as a declarative statement or a recommendation of action. In this measurement systems evaluation expert system, however, statements in the form of "attributepredicate-value" represent both sides of a rule, and both sides may include supporting procedures. These procedures are executable program routines implemented as part of the inference engine. If a procedure is to be used in the condition part of a rule, it is called a FUNCTION because it can be evaluated to be either true or false. If a procedure is to be used in the conclusion part of a rule then it is called a PROCEDURE because it will actually perform a predefined process.

Figure 1 shows a small portion of the rules for conducting a measurement systems evaluation study. 
The set of rules indicates the condition of the gauging equipment to be used in planed quality assurance studies. For this rule base, the gauge will be classified as "acceptable", "may be acceptable", or "generally not acceptable", depending upon its computation result of gauge error percent. Also, if a long method of gauging R\&R study is performed, a comparison of the measurement system's reproducibility error and repeatability error will be made in determining the possible causes of gauging error. The parameter values used in the rules are obtained from open literature $[1,5,10,11,12]$ or field experts. For example, Rule \#6 says that if the gauge error percent is greater than $30 \%$, then this gauge is generally not acceptable for its intended application. This rule also triggers Rule \#7 and Rule \#8 so the gauge error causes can be determined.

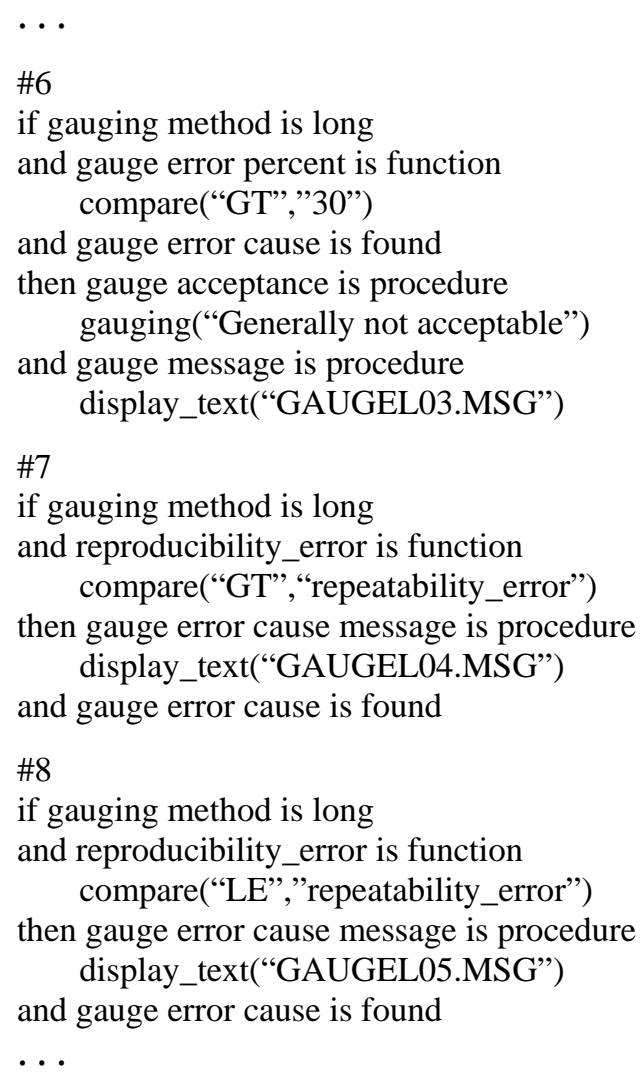

Figure 1.Sample Rules for Gauging Evaluation

\section{DEMONSTRATION AND EVALUATION}

The expert system developed in the previous section represents a knowledge-based methodology and platform for comprehensive measurement systems evaluation. The purpose of this section is to demonstrate and to evaluate the efficiency and effectiveness of the expert system in aiding quality assurance personnel in conducting such a gauging R\&R study.

How to evaluate an expert system is a complex question. A variety of methods and criteria may be considered. Gaschnig et al. have identified five characteristics of the expert system to evaluate. They are: (1) the quality of the system's decisions and advice, (2) the correctness of the reasoning techniques used, (3) the quality of the humancomputer interaction, (4) the system's efficiency, and (5) its cost effectiveness [8]. These five evaluation criteria will be broadly examined in relationship to the expert system developed for this study.

To accomplish this, the section begins by presenting a sample session with the expert system. This demonstration will manifest the system flow and how a gauging R\&R study team can benefit from this guided tour approach.

\section{A Sample Session with the Expert System}

The developed measurement systems evaluation expert system runs on a Windows-based personal computer with minimum system requirements. In order to demonstrate the expert system's capability in guiding a gauging $\mathrm{R} \& \mathrm{R}$ study, a series of screens generated in an actual consultation session will be presented. Because of space limitations, it will not be possible to reproduce every display. However, enough will be shown to convey the way a study team would typically interact with the system.

The display screens incorporated in the system all rely on a common graphic user interface that divides a screen into three areas. The top portion of the screen is the title and menu section that lists modules and functions that may be invoked from the current location in the system. The middle division of the screen is the consultation dialogue section that to be used for soliciting user input and displaying system messages. The bottom portion of the screen has all the command options that can be exercised in conjunction with the current display.

The system's opening screen that shows the main modules in the system appears upon the program starts. The menu consists of five items that cover the entire range of measurement systems evaluation process. One of the entries from the menu may be "pressed down," indicating that it is the active procedure selected by the user. The menu section also includes three buttons for system level activitiesHelp, Print, and Exit. 
The first procedure of the system is "Gauge". The user uses this module in defining the measurement system to be studied. In this screen, the quality assurance personnel will enter the name of the gauge, the name of the part, as well as all related parameters and specifications that need documentation.

The expert system provides some substantial assistance in conducting a gauging study. Figure 2 shows the Gauging R\&R Assessment provided by the system for preparing the user in conducting a meaningful measurement systems evaluation study. The user's input to the assessment questionnaire is then evaluated and appropriate suggestions are displayed in a subsequent screen.

The next step in gauging study is "Method". Through this module, the user can choose an appropriate method to use in the gauge R\&R study. Figure 3 shows that the Long Method is selected along with the display of a description of the chosen method. If the user needs more information regarding the exact procedure of performing such a study, the "Note" command shown at the left-bottom corner of the screen will activate the system's context-sensitive help facility to display a subject-specific tutorial.
Figure 4 shows the system's spreadsheet-like data entry screen for the gauging R\&R study with a set of observations entered. After the data set has been entered, it is analyzed by the system automatically. The appraisal outcome and relevant expert advice drawn from the system's knowledge base are then displayed in the "Result" module. Figure 5 shows the gauge R\&R study results, and Figure 6 shows possible causes and interpretation of gauge errors. After the completion of the measurement systems evaluation, the study team needs to analyze and document the results. If the degree of gauging error is unacceptable, every effort should be made to identify the problem and get it corrected before proceeding with the next step in machine/process qualification study or statistical process control.

Once a measurement systems evaluation is completed, a hard copy report of the study can be generated by using the "Print" button from the menu. A Gauging Study Report consists of information about the gauge, the part, the machine, as well as details of the measurements, the computation, the gauging $R \& R$ analysis results, and conclusions/recommendations.

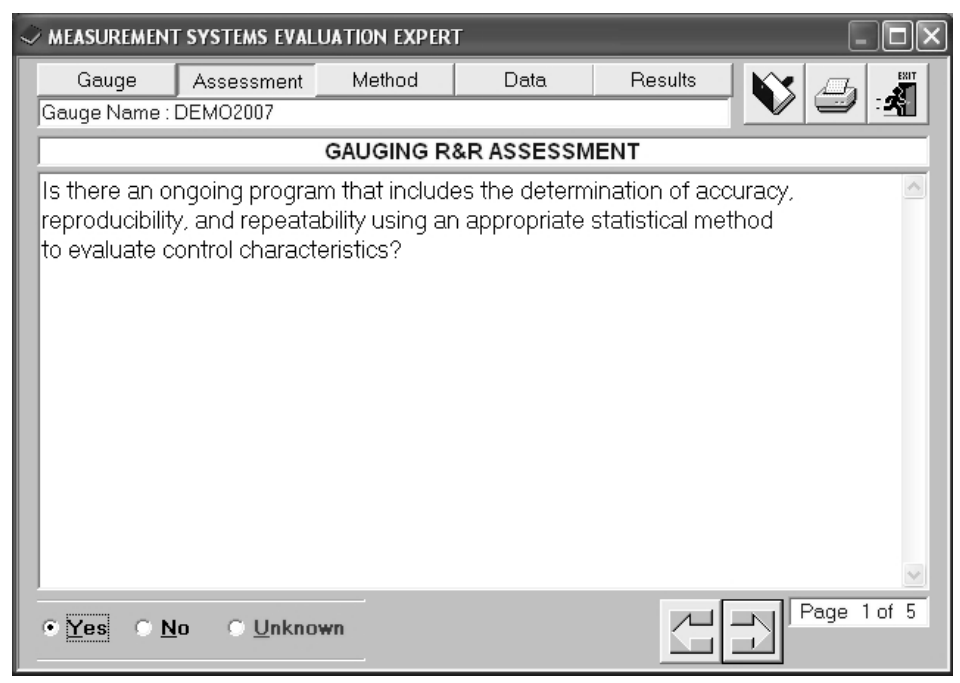

Figure 2. Gauging R\&R Assessment 


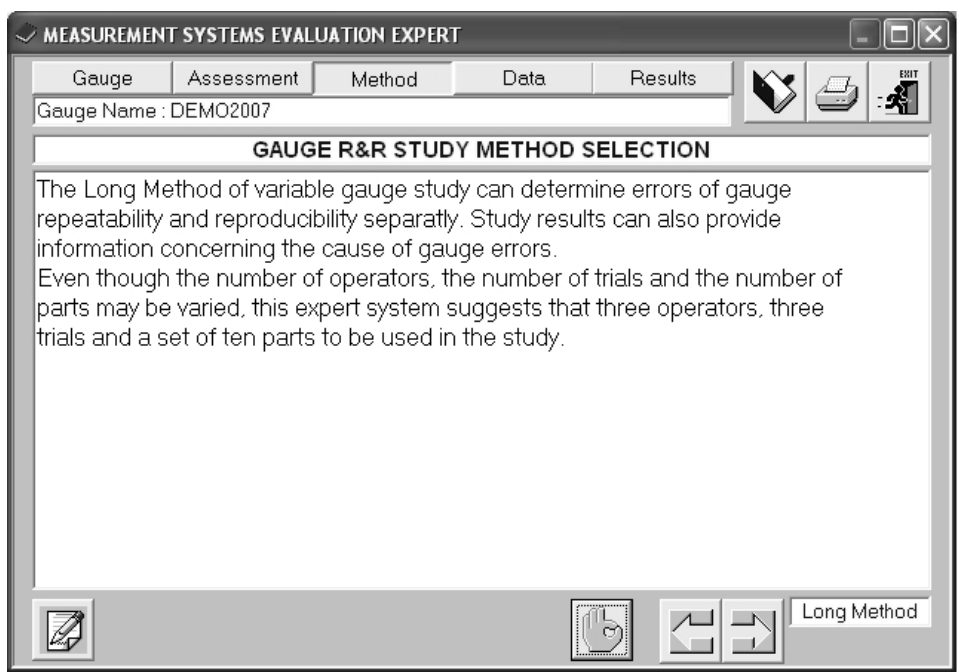

Figure 3. Gauging R\&R Study Method Selection

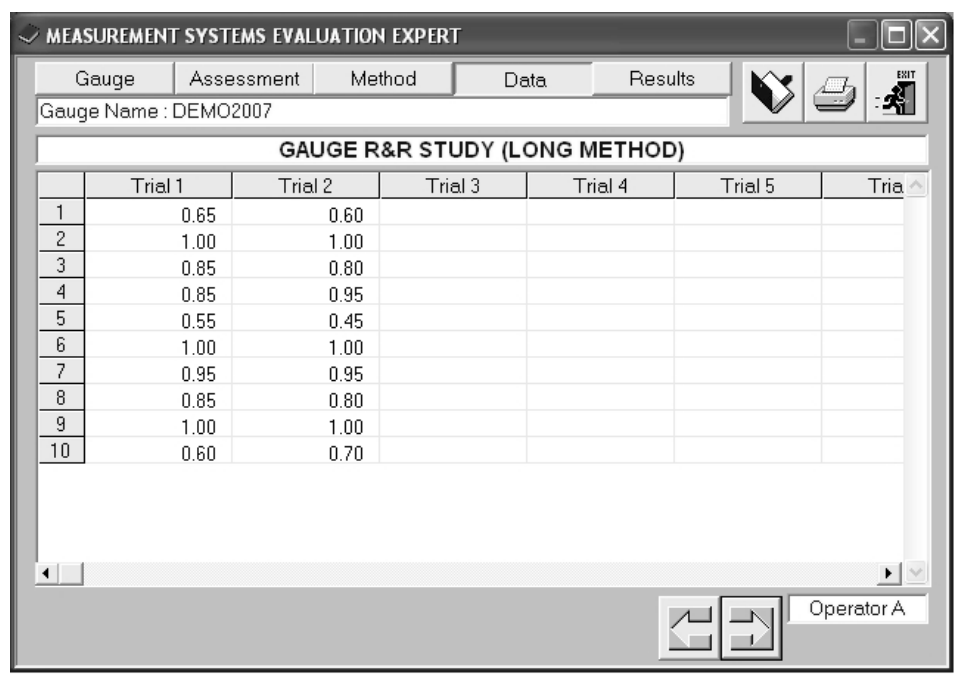

Figure 4. Gauging Study Date Entry Worksheet

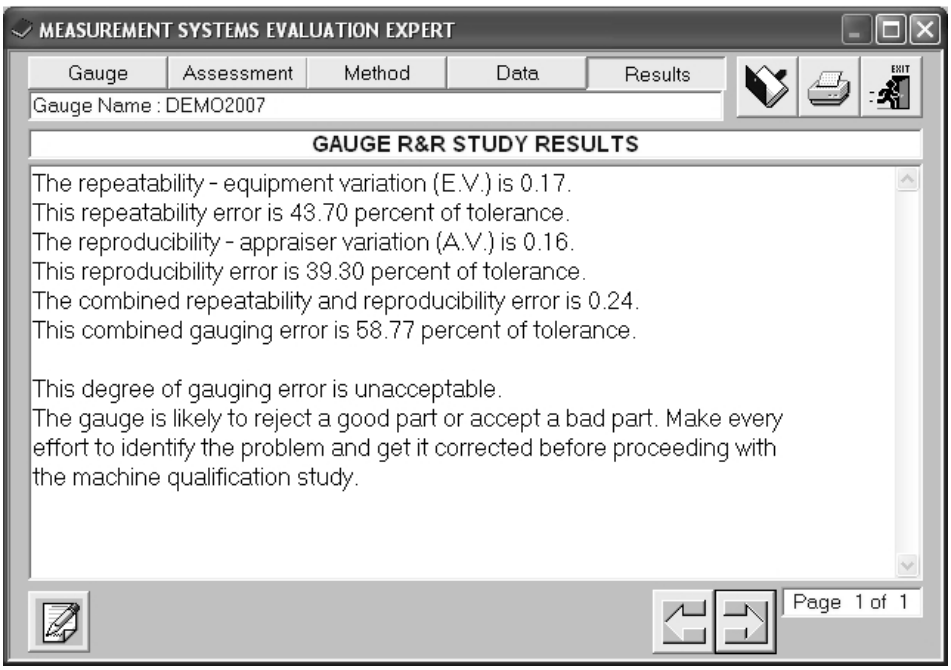

Figure 5. Gauging R\&R Study Results 


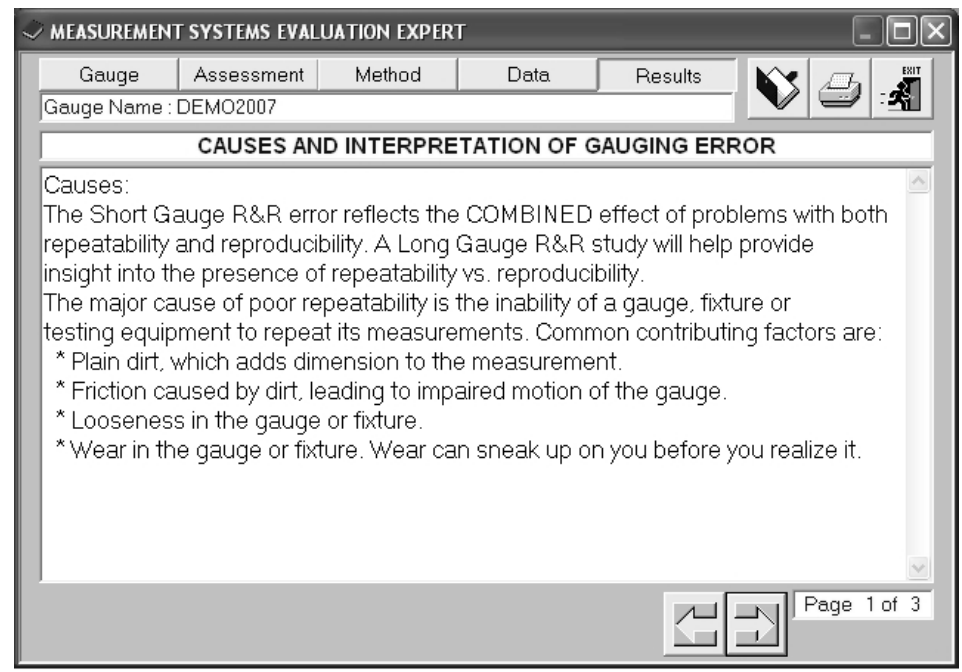

Figure 6. Causes and Interpretation of Gauging Error

\section{System Performance and Evaluation}

The measurement systems evaluation expert system has been field tested by quality engineers in several auto parts assembly plants during and after the initial development. Their preliminary findings are very positive. Many of the expert system's characteristics and implementation ideas in fact are drawn from their feedback. As compared to the conventional manual approach for conducting such studies, the quality engineers who tested this system point out that the expert system has the following noteworthy features and benefits:

1. The ability to link various steps of a gauging R\&R study together, and to provide gateways to control the flow of the study.

2. The ability to incorporate company policy and procedures into the evaluation effort (such as not allowing any other machine/process qualification study to proceed if the proper gauging calibration has not been performed and certified).

3. Improved accuracy of the gauging $R \& R$ study through more objective diagnostic interpretations and conclusions of evaluation data.

4. Reduced time to conduct a study.

5. Reduced needs for training shop personnel in statistical quality control methodology.

However, experience has also shown that the system is not without its limitations. The most apparent deficiency of the system is in incorporating logic to duplicate the experience and judgment necessary in analyzing and interpreting gauge-specific problems. For instance, the expert system's knowledge base currently contains only a collection of generic findings and rules in diagnosing gauging errors and suggesting corrective actions. It would be a great enhancement if the system could incorporate and utilize individualized knowledge bases tailored to a given gauge to diagnose and interpret specific tooling or material-related causes of problems.

Literature on information systems implementation suggests that one measure of success for a voluntary system is use [9]. The measurement systems evaluation expert system developed in this research is clearly a voluntary system, since it contains mostly existing methodology for gauging R\&R analysis in its entirety. When the system was being field tested at production plants, the quality assurance personnel could simply give it a sparse review without any serious use or investigation. However, they did use the system with enthusiasm and provided a great deal of feedback during the experimental stage. This fact, again, evidenced that the development and implementation of this knowledge-based expert system approach can truly be a success.

\section{SUMMARY AND REMARKS}

The goal of this research was to develop an expert system for measurement systems evaluation. This goal was accomplished and led to many discoveries about the difficulties and problems in gauging qualification practice as well as expert systems implementation. This final section of the paper will provide a summary of the measurement systems evaluation expert system methodology and platform, the accomplishments and findings result from this research. It will also present a cursory discussion on the possible improvements of the expert system, as well as potential directions for further research.

\section{Summary}


This paper has described and demonstrated a knowledge-based expert system approach for conducting measurement systems evaluation. The approach presented here provides a comprehensive, computerized system that efficiently and effectively guides the quality assurance team through such a study. Consequently, the results from the study will be more accurate and informative. Furthermore, since the entire evaluation methodology is integrated with an expert system, this new knowledge-based methodology and platform greatly enhanced the power of its utilization.

The heart of the measurement systems evaluation expert system is its inference engine and the knowledge base. The system's rule-based backwardchaining inference engine has a distinctive feature. That is, the inference engine has the ability to invoke system built-in numeric computation procedures. The knowledge base, on the other hand, provides the system with heuristic rules so that the inference engine can effectively interpret the test results and provide diagnosis and recommendations to the user.

The success of this approach suggests that integrating symbolic and numeric models is an excellent way to support decision-makers. The numeric model has its strength in clearly expressing a theoretical solution. The symbolic model makes it possible to adjust a theoretical solution to a specific problem domain. Therefore, a design of expert system with this type of integration can actually enjoy the strengthened interface between theory and reality.

Given all of the arguments above, it seems safe to conclude that this research was successful in developing a knowledge-based methodology and platform for measurement systems evaluation. The system developed represents an implementation of the methodology. During the field testing phase in various auto parts plants, the system effectively guided the user in conducting comprehensive measurement systems evaluation studies and functioned successfully in diagnosing gauging $R \& R$ related problems, as well as giving appropriate suggestions at the time the user expected to receive some expert advice.

\section{Remarks and Suggestions}

The applicability and importance of expert systems in industry has been widely acknowledged in words and in actions. Expert systems are the kind of tools that improve the productivity of professional workers, where performance levels that equal to or surpass human levels can be achieved. With expert systems technology, scarce knowledge and expertise can be captured, represented, packaged, and distributed. Automated systems can be developed that will benefit from this captured knowledge by providing better operations and additional aids to the systems' intended users.

As with any study of this nature, there are diverse tracks that future research could be directed. These directions can either be related to the measurement systems evaluation methodology used in the expert system or the implementation issues of expert systems technology in general.

The examination of existing statistical quality control and process capability analysis techniques provided a view of some of the basics of measurement systems evaluation, but it did not provide enough detail in some places, especially for the causes and interpretations of gauging error. An in-depth examination of certain measurement systems evaluation methodology would be worthwhile. To achieve the necessary depth, a comprehensive personal interview of field experts would be more desirable. Open literature usually does not provide the needed insight into the utilization of the various gauges or measuring techniques, especially on how to handle exceptions. The interviews should come from a variety of different manufacturing environments in order to gain the necessary coverage of diverse measuring and evaluation situations. To complete such an undertaking would require a tremendous amount of time, financial support, and cooperation of the people involved in the manufacturing quality assurance area.

This study has demonstrated the potential effectiveness and improved efficiency result from this integration, and laid the framework for utilizing knowledge-based measurement systems evaluation techniques. It has also pointed out directions for further research. The development effort and technological advancement of these areas of future research will certainly determine if a knowledgebased manufacturing systems qualification and diagnostic methodology and a truly automated online machine/process quality monitoring expert system will present in future manufacturing facilities.

\section{REFERENCES}

1. American Society for Quality Control. Glossary and Tables for Statistical Quality Control. Milwaukee, Wisconsin: American Society for Quality Control, 1983. 
2. CLIPS: A Tool for Building Expert Systems. WWW Online Document, Internet. Available: http:/ www.ghg.net/clips/CLIPS.html.

3. Connell, L. W. "Quality at the Source: The First Step in Just-in-Time Production." Quality Progress 17, no. 11 (November 1984): 44-45.

4. EXSYS, Inc. EXSYS Product Description. WWW Online Document, Internet. Available: http:// www.exsys.com.

5. General Motors Corporation. General Motors Statistical Process Control Manual. General Motors Corporation, 1986.

6. General Motors Corporation. General Motors Manufacturing Systems Qualification Manual. General Motors Corporation, 1987.

7. General Motors Corporation. Rochester Products Division. Machine Qualification Manual. Rochester, New York: Rochester Products Division, GMC, 1983.

8. Gasching J., P. Klahr, H. Pople, E. Shortliffe, and A. Terry. "Evaluation of Expert Systems:
Issues and Case Studies,” in Hayes-Roth, F., D. Waterman, and D. Lenat, eds. Building Expert Systems. Reading, Massachusetts: AddisonWesley Publishing Company, 1983.

9. Jackson, P. Introduction to Expert Systems. Wokingham, England: Addison-Wesley Publishing Company, 1986.

10. Juran, J. M. and F. M. Gryna, eds. Quality Control Handbook. 4th ed., New York, New York: McGraw-Hill Book Company, Inc., 1988.

11. McCaslin, J. A. and G. F. Gruska. "Analysis of Attribute Gage Systems." In 1976 ASQC Annual Technical Conference Transactions. American Society for Quality Control, 1976, 392-399.

12. Wheeler, D. J. and R. W. Lyday. Evaluating the Measurement Process. 2d ed., Knoxville, Tennessee: Statistical Process Controls, Inc., 1989. 